\title{
LIMIT THEOREMS FOR DECOMPOSABLE BRANCHING PROCESSES IN A RANDOM ENVIRONMENT
}

\author{
VLADIMIR VATUTIN, ${ }^{*}$ Steklov Mathematical Institute \\ QUANSHENG LIU,** Université de Bretagne-Sud
}

\begin{abstract}
We study the asymptotics of the survival probability for the critical and decomposable branching processes in a random environment and prove Yaglom-type limit theorems for these processes. It is shown that such processes possess some properties having no analogues for the decomposable branching processes in a constant environment
\end{abstract}

Keywords: Decomposable branching processes; survival probability; random environment

2010 Mathematics Subject Classification: Primary 60J80

Secondary 60F17; 60J85

\section{Introduction}

The multitype branching processes in a random environment we consider here can be viewed as a discrete-time stochastic model for the sizes of a geographically structured population occupying islands labeled $0,1, \ldots, N$. One unit of time represents a generation of particles (individuals). Particles located on island 0 give birth under the influence of a randomly changing environment. They may migrate to one of the islands $1,2, \ldots, N$ immediately after birth with probabilities again depending upon the current environmental state. Particles of island $i \in\{1,2, \ldots, N-1\}$ either stay at the same island or migrate to the islands $i+1,2, \ldots, N$ and their reproduction laws are not influenced by any changing environment. Finally, particles of island $N$ do not migrate and continue to evolve in a constant environment.

The goal of this paper is to investigate the asymptotic behavior of the survival probability of the whole process and the distribution of the number of particles in the population given its survival or survival of particles of type 1 .

Let $m_{i, j}$ be the mean number of type $j$ particles produced by a type $i$ particle at her death and $Z_{n, j}$ be the number of type $j$ particles in the process at moment $n$. Sometimes we will write $m_{i j}$ and $Z_{n j}$ for $m_{i, j}$ and $Z_{n, j}$, respectively, if there is no confusion.

We formulate our main assumptions as follows.

Assumption 1. (i) Particles of type 0 form (on their own) a critical branching process in a random environment.

(ii) Particles of any type $i \in\{1,2, \ldots, N\}$ form (on their own) a critical branching process in a constant environment, i.e. $m_{i, i}=1$.

Received 7 March 2014; revision received 25 August 2014.

* Postal address: Steklov Mathematical Institute, Gubkin str. 8, 119991, Moscow, Russia.

Email address: vatutin@mi.ras.ru

** Postal address: Université de Bretagne-Sud, UMR 6205, LMBA, F-56000 Vannes, France. 
(iii) Particles of any type $i$ are able to produce descendants of all the next in order types (may be not as the direct descendants) but not any preceding ones. In particular, $m_{i, j}=0$ for $1 \leq j<i \leq N$ and $m_{i, i+1}>0$ for $i=1, \ldots, N-1$.

Let $X_{n}$ be the number of particles of type 0 and $Z_{n}=\left(Z_{n, 1}, \ldots, Z_{n, N}\right)$ be the vector of the numbers of particles type $1,2, \ldots, N$, respectively, present at time $n$. Throughout this paper, considering the $(N+1)$-type branching process, it is assumed (unless otherwise specified) that $X_{0}=1$ and $Z_{0}=(0, \ldots, 0)=\mathbf{0}$.

We investigate asymptotics of the survival probability of this process as $n \rightarrow \infty$ and the distribution of the number of particles in the process at moment $n$ given $Z_{n 1}>0$ or $\boldsymbol{Z}_{n} \neq \mathbf{0}$. Note that the asymptotic behavior of the survival probability for the $N=1$ case has been investigated in [9] under stronger assumptions than those imposed in this paper. The essential novelty of this paper are Yaglom-type limit theorems for the population vector $\boldsymbol{Z}_{n}$ (see Theorem 2 below).

The structure of the remaining part of this paper is as follows. In Section 2 we recall known facts for decomposable branching processes in constant environments and present some preliminary results. In Section 3 we deal with the $(N+1)$-type decomposable branching processes in a random environment. Here, we study the asymptotic behavior of the survival probability and prove a Yaglom-type conditional limit theorem for the number of particles in the process given $Z_{n 1}>0$. In Section 4 we consider a 3-type decomposable branching process in a random environment and, proving a Yaglom-type conditional limit theorem under the condition $Z_{n 1}+Z_{n 2}>0$ show the essential difference of such processes with the decomposable processes evolving in a constant environment.

\section{Multitype decomposable branching processes in a constant environment}

The aim of this section is to present a number of known results about the decomposable branching processes. We are interested in the case of a constant environment and, therefore, we do not deal with particles of type 0 .

If Assumption 1 holds then the mean matrix of our process has the form

$$
\boldsymbol{M}=\left(m_{i j}\right)=\left(\begin{array}{ccccc}
1 & m_{1,2} & \ldots & \ldots & m_{1, N} \\
0 & 1 & m_{2,3} & \ldots & m_{2, N} \\
0 & 0 & 1 & \ldots & \cdots \\
\cdots & \ldots & \ldots & \cdots & \ldots \\
\cdots & \ldots & \ldots & \ldots & m_{N-1, N} \\
0 & 0 & \ldots & 0 & 1
\end{array}\right)
$$

where

$$
m_{i, i+1}>0, \quad i=1,2, \ldots, N-1 .
$$

Under conditions (1) and (2) one obtains a complete ordering $1 \rightarrow 2 \rightarrow \cdots \rightarrow N$ of types.

Observe that according to the classification given in [6] the process we consider is strongly critical.

In what follows, we need some results from [5] and [6]. To this aim, we introduce additional notation.

1. For any vector $s=\left(s_{1}, \ldots, s_{p}\right)$ (the dimension will usually be clear from the context), and integer-valued vector $\boldsymbol{k}=\left(k_{1}, \ldots, k_{p}\right)$ define

$$
\boldsymbol{s}^{k}=s_{1}^{k_{1}} \cdots s_{p}^{k_{p}} .
$$


Furthermore, let $\mathbf{1}=(1, \ldots, 1)$ be a vector of units and let $\boldsymbol{e}_{i}$ be a vector whose $i$ th component is equal to 1 while the remaining are 0 .

2. The first and second moments of the components of the population vector $Z_{n}=$ $\left(Z_{n 1}, \ldots, Z_{n N}\right)$ will be denoted as

$$
\begin{gathered}
m_{i l}(n):=\boldsymbol{E}\left[Z_{n l} \mid Z_{0}=\boldsymbol{e}_{i}\right], \quad m_{i l}:=m_{i l}(1), \\
b_{i k l}(n):=\boldsymbol{E}\left[Z_{n k} Z_{n l}-\delta_{k l} Z_{n l} \mid \boldsymbol{Z}_{0}=\boldsymbol{e}_{i}\right], \quad b_{i k l}:=b_{i k l}(1),
\end{gathered}
$$

where $\boldsymbol{E}$ is the expectation in a constant environment.

To go further, we introduce probability generating functions

$$
h^{(i, N)}(s):=E\left[\prod_{k=i}^{N} s_{k}^{\eta_{i k}}\right], \quad 1 \leq i \leq N,
$$

where $\eta_{i j}$ represents the number of children of type $j$ of a parent of type $i \in\{1,2, \ldots, N\}$. Let

$$
H_{n}^{(i, N)}(s):=\boldsymbol{E}\left[\prod_{k=i}^{N} s_{k}^{Z_{n k}} \mid \boldsymbol{Z}_{0}=\boldsymbol{e}_{i}\right], \quad 1 \leq i \leq N,
$$

be the probability generating functions for the vector of the number of particles at moment $n$ given the process is initiated at time 0 by a single particle of type $i \in\{1,2, \ldots, N\}$. Clearly, $H_{1}^{(i, N)}(s)=h^{(i, N)}(s)$. Denote

$$
\begin{gathered}
\boldsymbol{H}_{n}(\boldsymbol{s}):=\left(H_{n}^{(1, N)}(\boldsymbol{s}), \ldots, H_{n}^{(N, N)}(\boldsymbol{s})\right), \\
\boldsymbol{Q}_{n}(\boldsymbol{s}):=\left(Q_{n}^{(1, N)}(\boldsymbol{s}), \ldots, Q_{n}^{(N, N)}(s)\right)=\left(1-H_{n}^{(1, N)}(s), \ldots, 1-H_{n}^{(N, N)}(s)\right) .
\end{gathered}
$$

As usual, for two sequences $a_{n}, b_{n}$, we write $a_{n} \sim b_{n}, a_{n}=O\left(b_{n}\right), a_{n}=o\left(b_{n}\right)$, and $a_{n} \asymp b_{n}$ meaning that these relationships are valid as $n \rightarrow \infty$. In particular, $a_{n} \asymp b_{n}$ if and only if

$$
0<\liminf _{n \rightarrow \infty} \frac{a_{n}}{b_{n}} \leq \limsup _{n \rightarrow \infty} \frac{a_{n}}{b_{n}}<\infty .
$$

The following theorem is a simplified combination of the respective results from [5] and [6].

Theorem 1. (i) Let $\left\{Z_{n}, n=0,1, \ldots\right\}$ be a strongly critical multitype branching process satisfying (1) and (2). Then, as $n \rightarrow \infty$,

$$
m_{i l}(n) \sim c_{i l} n^{l-i}, \quad i \leq l,
$$

where $c_{i l}$ are positive constants known explicitly (see [6, Theorem 1]);

(ii) if $b_{i k l}<\infty, i, k, l=1, \ldots, N$ then

$$
b_{i k l}(n) \sim c_{i k l} n^{k+l-2 i+1},
$$

where $c_{i k l}$ are constants known explicitly (see [6, Theorem 1]) and

$$
Q_{n}^{(i, N)}(\mathbf{0})=1-H_{n}^{(i, N)}(\mathbf{0})=\boldsymbol{P}\left(\boldsymbol{Z}_{n} \neq \mathbf{0} \mid \boldsymbol{Z}_{0}=\boldsymbol{e}_{i}\right) \sim c_{i} n^{-2^{-(N-i)}}, \quad c_{i}>0,
$$

where $\boldsymbol{P}$ is the probability measure in a constant environment. 
Let $H\left(s_{1}, \ldots, s_{p}\right)=H(s)$ be a multivariate probability generating function with

$$
m_{l}:=\left.\frac{\partial H(s)}{\partial s_{l}}\right|_{s=1}, \quad b_{k l}:=\left.\frac{\partial^{2} H(s)}{\partial s_{k} \partial s_{l}}\right|_{s=1}<\infty .
$$

Lemma 1. (See [3, Equation (1), p. 189].) For any $s=\left(s_{1}, \ldots, s_{p}\right) \in[0,1]^{p}$, we have

$$
\sum_{l=1}^{p} m_{l}\left(1-s_{l}\right)-\frac{1}{2} \sum_{k, l=1}^{p} b_{k l}\left(1-s_{k}\right)\left(1-s_{l}\right) \leq 1-H(s) \leq \sum_{l=1}^{p} m_{l}\left(1-s_{l}\right) .
$$

From now on, we denote by $C, C_{0}, C_{1}, \ldots$ positive constants which may be different in different equations.

For $s=\left(s_{1}, \ldots, s_{N}\right)$ put

$$
M_{i}(n ; s):=\sum_{l=i}^{N} m_{i l}(n)\left(1-s_{l}\right), \quad B_{i}(n ; s):=\frac{1}{2} \sum_{k, l=i}^{N} b_{i k l}(n)\left(1-s_{k}\right)\left(1-s_{l}\right) .
$$

Lemma 2. Let the conditions of Theorem 1 hold. Then for any tuple $t_{1}, \ldots, t_{N}$ of positive numbers and

$$
1-s_{l}=n^{-t_{l}}, \quad l=1,2, \ldots, N,
$$

there exists $C_{+}<\infty$ such that, for all $n=1,2, \ldots$,

$$
Q_{n}^{(i, N)}(\boldsymbol{s}) \leq C_{+} \min \left\{n^{-2^{-(N-i)}}, n^{-\min _{i \leq l \leq N}\left(t_{l}-l+i\right)}\right\} .
$$

If, in addition,

$$
\min _{i \leq l \leq N}\left(t_{l}-l+i\right) \geq 1
$$

then there exists a positive constant $C_{-}$such that, for all $n=1,2, \ldots$,

$$
C_{-} n^{-\min _{i \leq l \leq N}\left(t_{l}-l+i\right)} \leq Q_{n}^{(i, N)}(s) \leq C_{+} n^{-\min _{i \leq l \leq N}\left(t_{l}-l+i\right)} .
$$

Proof. Take $\varepsilon \in(0,1]$ and denote $s(\varepsilon)=\left(1-\varepsilon n^{-t_{1}}, \ldots, 1-\varepsilon n^{-t_{N}}\right)$. By Lemma 1 and the monotonicity of $Q_{n}^{(i, N)}(s(\varepsilon))$ in $\varepsilon$, we have

$$
M_{i}(n ; s(\varepsilon))-B_{i}(n ; s(\varepsilon)) \leq Q_{n}^{(i, N)}(s(\varepsilon)) \leq Q_{n}^{(i, N)}(s) \leq M_{i}(n ; s) .
$$

In view of (4) and (5) there exist positive constants $C_{j}, j=1,2,3,4$ such that

$$
\begin{aligned}
{ }_{\varepsilon} C_{1} n^{-\min _{i \leq l \leq N}\left(t_{l}-l+i\right)} & \leq \varepsilon C_{1} \sum_{l=i}^{N} \frac{n^{l-i}}{n^{t_{l}}} \leq M_{i}(n ; s(\varepsilon))=\varepsilon \sum_{l=i}^{N} m_{i l}(n) n^{-t_{l}} \\
& \leq M_{i}(n ; s) \leq C_{2} \sum_{l=i}^{N} \frac{n^{l-i}}{n^{t_{l}}} \\
& \leq C_{3} n^{-\min _{i \leq l \leq N}\left(t_{l}-l+i\right)}
\end{aligned}
$$

and

$$
0 \leq B_{i}(n ; s(\varepsilon)) \leq \varepsilon^{2} C_{4} \sum_{k, l=i}^{N} \frac{n^{k-i+1+l-i}}{n^{t_{k}} n^{t_{l}}}
$$


If $\min _{i \leq k \leq N}\left(t_{k}-k+i-1\right) \geq 0$ then for a fixed $\varepsilon>0$,

$$
0 \leq B_{i}(n ; s(\varepsilon)) \leq \varepsilon^{2} C_{4} \sum_{k, l=i}^{N} \frac{1}{n^{t_{l}-(l-i)} n^{t_{k}-(k-i+1)}} \leq \varepsilon^{2} N^{2} C_{4} n^{-\min _{i \leq l \leq N}\left(t_{l}-l+i\right)} .
$$

Take $0<\varepsilon<\min \left\{1, C_{1} / N^{2} C_{4}\right\}$. From the estimates (9)-(11), we obtain (8) with $C_{-}=$ ${ }_{\varepsilon} C_{1}-\varepsilon^{2} N^{2} C_{4}$ and $C_{+}=C_{3}$.

Write $\mathbf{0}^{(r)}=(0,0, \ldots, 0)$ and $\mathbf{1}^{(r)}=(1,1, \ldots, 1)$ for the $r$-dimensional vectors whose components are all 0 and 1, respectively; set $\boldsymbol{s}_{r}=\left(s_{r}, s_{r+1}, \ldots, s_{N}\right)$ and denote by $\mathbf{1}_{\{\mathcal{A}\}}$ the indicator of the event $\mathcal{A}$.

In the next lemma, we assume that $\boldsymbol{Z}_{0}=\boldsymbol{e}_{1}$ and provide an approximation for the function $Q_{n}^{(1, N)}\left(\mathbf{0}^{(r)}, \boldsymbol{s}_{r+1}\right)$.

Lemma 3. If $\min _{r+1 \leq l \leq N}\left(t_{l}-l+1\right)>2^{-(r-1)}$ and

$$
1-s_{l}=n^{-t_{l}}, \quad l=r+1, r+2, \ldots, N,
$$

then, as $n \rightarrow \infty$,

$$
Q_{n}^{(1, N)}\left(\mathbf{0}^{(r)}, \boldsymbol{s}_{r+1}\right) \sim \boldsymbol{P}\left(Z_{n r}>0\right) \sim c_{r} n^{-2^{-(r-1)}} .
$$

Proof. In view of (6) for $s_{r+1} \in[0,1]^{N-r}$, we have

$$
\begin{aligned}
\boldsymbol{P}\left(Z_{n r}>0\right) & \leq \boldsymbol{P}\left(\bigcup_{j=1}^{r}\left\{Z_{n j}>0\right\}\right) \\
& =Q_{n}^{(1, N)}\left(\mathbf{0}^{(r)}, \mathbf{1}^{(N-r)}\right) \\
& \leq Q_{n}^{(1, N)}\left(\mathbf{0}^{(r)}, \boldsymbol{s}_{r+1}\right) \\
& =\boldsymbol{E}\left[1-s_{r+1}^{Z_{n, r+1}} \cdots s_{N}^{Z_{n N}} \mathbf{1}_{\left.\left\{\cap_{j=1}^{r}\left\{Z_{n j}=0\right\}\right\}\right]}\right] \\
& \leq \boldsymbol{P}\left(\bigcup_{j=1}^{r}\left\{Z_{n j}>0\right\}\right)+\boldsymbol{E}\left[1-s_{r+1}^{Z_{n, r+1}} \cdots s_{N}^{Z_{n N}}\right] \\
& \leq \sum_{j=1}^{r} \boldsymbol{P}\left(Z_{n j}>0\right)+\boldsymbol{E}\left[1-s_{r+1}^{Z_{n, r+1}} \cdots s_{N}^{\left.Z_{n N}\right]}\right. \\
& =(1+o(1)) \boldsymbol{P}\left(Z_{n r}>0\right)+Q_{n}^{(1, N)}\left(\mathbf{1}^{(r)}, \boldsymbol{s}_{r+1}\right) .
\end{aligned}
$$

Furthermore, by the conditions of the lemma, we deduce that

$$
\begin{aligned}
Q_{n}^{(1, N)}\left(\mathbf{1}^{(r)}, \boldsymbol{s}_{r+1}\right) & \leq \sum_{l=r+1}^{N} m_{1 l}(n) n^{-t_{l}} \\
& \leq C n^{-\min _{r+1 \leq l \leq N}\left(t_{l}-l+1\right)}=o\left(n^{-2^{-(r-1)}}\right) .
\end{aligned}
$$

Hence, the statement of the lemma follows. 


\subsection{The case of two types}

Here we consider the situation of two types and investigate the behavior of the function $1-H_{n}^{(1,2)}\left(s_{1}, s_{2}\right)$ as $n \rightarrow \infty$ assuming that $1-s_{i}=n^{-t_{i}}$ for $i=1,2$.

Lemma 4. If the conditions of Theorem 1 hold for $N=2$, then

$$
1-H_{n}^{(1,2)}\left(s_{1}, s_{2}\right) \asymp \begin{cases}n^{-1 / 2} & \text { if } t_{1} \in(0, \infty), 0<t_{2} \leq 1, \\ n^{-t_{2} / 2} & \text { if } t_{1} \in(0, \infty), 1<t_{2}<2, \\ n^{-1} & \text { if } 0<t_{1}<1, t_{2} \geq 2, \\ n^{-1-\min \left(t_{1}-1, t_{2}-2\right)} & \text { if } t_{1} \geq 1, t_{2} \geq 2 .\end{cases}
$$

Proof. Observe that, for any $0 \leq s_{1} \leq s_{1}^{\prime} \leq 1$,

$$
\begin{aligned}
H_{n}^{(1,2)}\left(s_{1}^{\prime}, s_{2}\right)-H_{n}^{(1,2)}\left(s_{1}, s_{2}\right) & =\boldsymbol{E}\left[\left(\left(s_{1}^{\prime}\right)^{Z_{n 1}}-s_{1}^{Z_{n 1}}\right) s_{2}^{Z_{n 2}}\right] \\
& \leq \boldsymbol{E}\left[1-s_{1}^{Z_{n 1}}\right] \\
& =1-H_{n}^{(1,1)}\left(s_{1}\right) \\
& \leq \boldsymbol{P}\left(Z_{n 1}>0 \mid \boldsymbol{Z}_{0}=\boldsymbol{e}_{1}\right) \\
& \leq C n^{-1}
\end{aligned}
$$

Now let $m=m\left(s_{2}\right)$ be specified by the inequalities

$$
Q_{m}^{(2,2)}(0) \leq 1-s_{2}=n^{-t_{2}} \leq Q_{m-1}^{(2,2)}(0)
$$

In view of

$$
Q_{m}^{(2,2)}(0)=1-H_{m}^{(2,2)}(0)=\boldsymbol{P}\left(Z_{m 2}>0 \mid \boldsymbol{Z}_{0}=\boldsymbol{e}_{2}\right) \sim \frac{2}{m \operatorname{var} \eta_{22}},
$$

it follows that $m \sim 2 n^{t_{2}} / \operatorname{var} \eta_{22}$. Using this fact, (12), and the branching property

$$
H_{n}^{(1,2)}\left(H_{m}^{(1,2)}(\boldsymbol{s}), H_{m}^{(2,2)}\left(s_{2}\right)\right)=H_{n+m}^{(1,2)}(s),
$$

by (6), we conclude that

$$
\begin{aligned}
1-H_{n}^{(1,2)}\left(s_{1}, s_{2}\right) & \geq 1-H_{n}^{(1,2)}\left(s_{1}, H_{m}^{(2,2)}(0)\right) \\
& =1-H_{n}^{(1,2)}\left(H_{m}^{(1,2)}(\mathbf{0}), H_{m}^{(2,2)}(0)\right)+O\left(n^{-1}\right) \\
& =Q_{n+m}^{(1,2)}(\mathbf{0})+O\left(n^{-1}\right) \\
& =(1+o(1)) c_{1}(n+m)^{-1 / 2}+O\left(n^{-1}\right) .
\end{aligned}
$$

Clearly, the result remains valid when ' $\geq$ ' is replaced by ' $\leq$ ' with $m$ replaced by $m-1$. Therefore, $1-H_{n}^{(1,2)}\left(s_{1}, s_{2}\right) \asymp n^{-1 / 2}$ if $t_{2} \in(0,1]$, and $1-H_{n}^{(1,2)}\left(s_{1}, s_{2}\right) \asymp n^{-t_{2} / 2}$ if $t_{2} \in(1,2)$. This proves the first two relationships of the lemma.

Consider now the $t_{2} \geq 2$ case. In view of (4),

$$
\begin{aligned}
1-H_{n}^{(1,1)}\left(s_{1}\right) & =1-H_{n}^{(1,2)}\left(s_{1}, 1\right) \\
& \leq 1-H_{n}^{(1,2)}\left(s_{1}, s_{2}\right) \\
& \leq 1-H_{n}^{(1,1)}\left(s_{1}\right)+n^{-t_{2}} \boldsymbol{E}\left[Z_{n 2} \mid \boldsymbol{Z}_{0}=\boldsymbol{e}_{1}\right] \\
& =1-H_{n}^{(1,1)}\left(s_{1}\right)+(1+o(1)) c_{12} n^{1-t_{2}} .
\end{aligned}
$$


Recalling that $1-s_{1}=n^{-t_{1}}$ and selecting $m=m\left(s_{1}\right)$ similar to (13), we obtain

$$
1-H_{n}^{(1,1)}\left(s_{1}\right) \sim 1-H_{n+m}^{(1,1)}(0) \asymp \frac{1}{n^{t_{1}}+n} .
$$

Hence, if $t_{1}<1$ then $1-H_{n}^{(1,2)}\left(s_{1}, s_{2}\right) \asymp n^{-1}$ as claimed.

The statement for $t_{1} \geq 1, t_{2} \geq 2$ follows from (8).

\section{Decomposable branching processes in a random environment}

The model of branching processes in a random environment which we are dealing with is a combination of the processes introduced by Smith and Wilkinson [8] and the ordinary decomposable multitype Galton-Watson processes. To provide a formal description of the model denote by $\mathcal{M}$ the space of probability measures on $\mathbb{N}_{0}^{N+1}$, where $\mathbb{N}_{0}:=\{0,1,2, \ldots\}$ and let $\mathfrak{e}$ be a random variable with values in $\mathcal{M}$. An infinite sequence $\mathcal{E}=\left(\mathfrak{e}_{1}, \mathfrak{e}_{2}, \ldots\right)$ of independent and identically distributed (i.i.d.) copies of $\mathfrak{e}$ is said to form a random environment.

We associate with $\mathfrak{e}$ and $\mathfrak{e}_{n}, n=1,2, \ldots$ random vectors $\left(\xi_{0}, \ldots, \xi_{N}\right)$ and $\left(\xi_{0}^{(n)}, \ldots, \xi_{N}^{(n)}\right)$ such that, for $\boldsymbol{k} \in \mathbb{N}_{0}^{N+1}$,

$$
\mathbb{P}\left(\left(\xi_{0}, \ldots, \xi_{N}\right)=\boldsymbol{k} \mid \mathfrak{e}\right)=\mathfrak{e}(\{\boldsymbol{k}\}), \quad \mathbb{P}\left(\left(\xi_{0}^{(n)}, \ldots, \xi_{N}^{(n)}\right)=\boldsymbol{k} \mid \mathfrak{e}_{n}\right)=\mathfrak{e}_{n}(\{\boldsymbol{k}\}) .
$$

We now specify a branching process $\left(X_{n}, Z_{n}\right)=\left(X_{n}, Z_{n 1}, \ldots, Z_{n N}\right)$ in a random environment $\mathcal{E}$ with types $0,1, \ldots, N$ as follows:

(i) $\left(X_{0}, Z_{0}\right)=(1, \mathbf{0})$;

(ii) Given $\mathcal{E}=\left(e_{1}, e_{2}, \ldots\right)$ and $\left(X_{n-1}, Z_{n-1}\right), n \geq 1$,

$$
X_{n}=\sum_{k=1}^{X_{n-1}} \xi_{k 0}^{(n-1)}, \quad Z_{n j}=\sum_{k=1}^{X_{n-1}} \xi_{k j}^{(n-1)}+\sum_{i=1}^{j} \sum_{k=1}^{Z_{(n-1) i}} \eta_{k, i j}^{(n-1)}, \quad j=1, \ldots, N,
$$

where the tuples $\left(\xi_{k 0}^{(n-1)}, \xi_{k 1}^{(n-1)}, \ldots, \xi_{k N}^{(n-1)}\right), k=1,2, \ldots, X_{n-1}$ are i.i.d. random vectors with distribution $e_{n-1}$, i.e. given $\mathfrak{e}_{n-1}=e_{n-1}$ distributed as $\left(\xi_{0}^{(n-1)}, \xi_{1}^{(n-1)}, \ldots\right.$, $\left.\xi_{N}^{(n-1)}\right)$, and the tuples $\left(\eta_{k i i}^{(n-1)}, \eta_{k i, i+1}^{(n-1)}, \ldots, \eta_{k i N}^{(n-1)}\right)$ are independent random vectors distributed as $\left(\eta_{i i}, \eta_{i, i+1}, \ldots, \eta_{i N}\right)$ for $i=1,2, \ldots N$, i.e. in accordance with the respective probability generating function $h^{(i, N)}(s)$ in (3).

Informally, $\xi_{k j}^{(n-1)}$ is the number of type $j$ children produced by the $k$ th particle of type 0 of generation $n-1$, while $\eta_{k, i j}^{(n-1)}$ is the number of type $j$ children produced by the $k$ th particle of type $i$ of generation $n-1$.

We denote by $\mathbb{P}$ and $\mathbb{E}$ the corresponding probability measure and expectation on the underlying probability space to distinguish them from the probability measure and expectation in a constant environment specified by $\boldsymbol{P}$ and $\boldsymbol{E}$.

Thus, in our model particles of type 0 belonging to the $(n-1)$ th generation give birth in total to $X_{n}$ particles of their own type and to the tuple $\boldsymbol{Y}_{n}=\left(Y_{n 1}, \ldots, Y_{n N}\right)$ of child particles of types $1,2, \ldots, N$, where

$$
Y_{n j}=\sum_{k=1}^{X_{n-1}} \xi_{k j}^{(n-1)}
$$

In particular, $\boldsymbol{Y}_{1}=\left(Y_{11}, \ldots, Y_{1 N}\right)=\left(\xi_{1}^{(0)}, \ldots, \xi_{N}^{(0)}\right)=Z_{1}$. 
Finally, each particle of type $i=1,2, \ldots, N$ generates its own (decomposable, if $i<N$ ) process with $N-i+1$ types evolving in a constant environment.

Let $\mu_{1}=\mathbb{E}\left[\xi_{0} \mid \mathfrak{e}\right], \mu_{2}=\mathbb{E}\left[\xi_{0}\left(\xi_{0}-1\right) \mid \mathfrak{e}\right]$, and

$$
\theta_{i}=\mathbb{E}\left[\xi_{i} \mid \mathfrak{e}\right], \quad i=1,2, \ldots, N, \quad \Theta_{1}:=\sum_{l=1}^{N} \theta_{l} .
$$

Our assumptions on the characteristics of the process we consider are formulated as follows.

Assumption 2. (i) The initial state of the process is $\left(X_{0}, Z_{0}\right)=(1,0)$.

(ii) Particles of type 0 form (on their own) a critical branching process in a random environment such that

$$
\mathbb{E} \log \mu_{1}=0, \quad \mathbb{E} \log ^{2} \mu_{1} \in(0, \infty) .
$$

(iii) Particles of type 0 produce particles of type 1 with a positive probability and

$$
\mathbb{P}\left(\theta_{1}>0\right)=1 \text {. }
$$

(iv) Particles of each type form (on their own) critical branching processes which are independent of the environment, i.e. $m_{i i}=\boldsymbol{E} \eta_{i i}=1, i=1,2, \ldots, N$.

(v) Particles of type $i=1,2, \ldots, N-1$ produce particles of type $i+1$ with a positive probability, i.e. $m_{i, i+1}=\boldsymbol{E} \eta_{i, i+1}>0, i=1,2, \ldots, N-1$.

(vi) The second moments of the offspring numbers are finite:

$$
\boldsymbol{E} \eta_{i j}^{2}<\infty, \quad 1 \leq i \leq j \leq N
$$

with $b_{i}=\frac{1}{2} \operatorname{var} \eta_{i i} \in(0, \infty)$.

The following theorem is the main result of the paper.

Theorem 2. If Assumption 2 holds and

$$
\mathbb{E}\left[\mu_{1}^{-1}\right]<\infty, \quad \mathbb{E}\left[\mu_{2} \mu_{1}^{-2}\left(1+\max \left(0, \log \mu_{1}\right)\right)\right]<\infty,
$$

then there exists a positive constant $K_{0}$ such that

$$
\mathbb{P}\left(\boldsymbol{Z}_{n} \neq 0 \mid X_{0}=1, \boldsymbol{Z}_{0}=\mathbf{0}\right) \sim \frac{2^{N-1} K_{0}}{\log n},
$$

and for any positive $t_{1}, t_{2}, \ldots, t_{N}$,

$$
\begin{aligned}
\lim _{n \rightarrow \infty} \mathbb{P}\left(\frac{\log Z_{n i}}{\log n} \leq t_{i}, i=1, \ldots, N \mid Z_{n 1}>0\right) & =G\left(t_{1}, \ldots, t_{N}\right) \\
& =1-\frac{1}{1+\max \left(0, \min _{1 \leq l \leq N}\left(t_{l}-l\right)\right)}
\end{aligned}
$$


The proof of the theorem is divided into several stages.

Let

$$
T=\min \left\{n \geq 0: X_{n}=0\right\} .
$$

According to [7, Theorem 1], if conditions (14) and (15) hold then for a positive constant $c$,

$$
\mathbb{P}\left(X_{n}>0\right)=\mathbb{P}(T>n) \sim \frac{c}{\sqrt{n}} \quad \text { as } n \rightarrow \infty
$$

Set $S_{n}:=\sum_{k=0}^{n-1} X_{k}$ and $A_{n}=\max _{0 \leq k \leq n-1} X_{k}$, so that $S_{\mathrm{T}}$ and $A_{\mathrm{T}}$ denote the total number ever born of type 0 particles and the maximal generation size of type 0 particles, respectively.

Lemma 5. (See [1].) If conditions (14) and (15) hold then there exists a constant $K_{0} \in(0, \infty)$ such that

$$
\mathbb{P}\left(S_{\mathrm{T}}>x\right) \sim \mathbb{P}\left(A_{\mathrm{T}}>x\right) \sim \frac{K_{0}}{\log x} \quad \text { as } x \rightarrow \infty .
$$

In fact, the representation (19) has been proved in [1] under conditions (14) and (15) only for the case when the probability generating functions of $\xi_{0}^{(n)}, n=0,1, \ldots$ are linear-fractional with probability 1 . However, this restriction is easily removed using the results established later on for the general case in [2] and [7].

Now let $\left\|\boldsymbol{Y}_{n}\right\|=Y_{n 1}+\cdots+Y_{n N}, \zeta_{k}^{(n)}=\xi_{k 1}^{(n-1)}+\cdots+\xi_{k N}^{(n-1)}$, and

$$
\begin{array}{ll}
L_{n j}=\sum_{l=1}^{n} Y_{l j}=\sum_{l=1}^{n} \sum_{k=1}^{X_{l-1}} \xi_{k j}^{(l-1)}, & B_{n j}=\max _{1 \leq l \leq n} Y_{l j}, \\
L_{n}=\sum_{l=1}^{n}\left\|\boldsymbol{Y}_{l}\right\|=\sum_{l=1}^{n} \sum_{k=1}^{X_{l-1}} \zeta_{k}^{(l-1)}, & B_{n}=\max _{1 \leq l \leq n}\left\|\boldsymbol{Y}_{l}\right\| .
\end{array}
$$

In particular, $L_{\mathrm{T}}$ is the total number of child particles of types $1, \ldots, N$ produced by type 0 particles during the evolution of the process.

Lemma 6. If conditions (14) and (15) hold and $\mathbb{P}\left(\Theta_{1}>0\right)=1$, then

$$
\mathbb{P}\left(B_{\mathrm{T}}>x\right) \sim \mathbb{P}\left(L_{\mathrm{T}}>x\right) \sim \frac{K_{0}}{\log x} \text { as } x \rightarrow \infty
$$

If conditions (15) and (14) hold and $\mathbb{P}\left(\theta_{j}>0\right)=1$ for some $j \in\{1, \ldots, N\}$, then

$$
\mathbb{P}\left(B_{\mathrm{T} j}>x\right) \sim \mathbb{P}\left(L_{\mathrm{T} j}>x\right) \sim \frac{K_{0}}{\log x} \quad \text { as } x \rightarrow \infty
$$

Proof. For any $\varepsilon \in(0,1)$, we have

$$
\mathbb{P}\left(A_{\mathrm{T}}>x\right) \leq \mathbb{P}\left(B_{\mathrm{T}}>x^{1-\varepsilon}\right)+\mathbb{P}\left(A_{\mathrm{T}}>x ; B_{\mathrm{T}} \leq x^{1-\varepsilon}\right) .
$$


Let $T_{x}=\min \left\{k: X_{k}>x\right\}$. Then

$$
\begin{aligned}
\mathbb{P}\left(A_{\mathrm{T}}>x ; B_{\mathrm{T}} \leq x^{1-\varepsilon}\right) & \leq \sum_{l=1}^{\infty} \mathbb{P}\left(T_{x}=l ;\left\|\boldsymbol{Y}_{l+1}\right\| \leq x^{1-\varepsilon}\right) \\
& =\sum_{l=1}^{\infty} \mathbb{P}\left(T_{x}=l ; \sum_{k=1}^{X_{l}} \zeta_{k}^{(l)} \leq x^{1-\varepsilon}\right) \\
& \leq \mathbb{P}\left(A_{\mathrm{T}}>x\right) \mathbb{P}\left(\sum_{k=1}^{[x]} \zeta_{k}^{(0)} \leq x^{1-\varepsilon}\right)
\end{aligned}
$$

Since $\mathbb{P}\left(\Theta_{1}>0\right)=1$ and $\Theta_{1}=\mathbb{E}\left[\zeta_{k}^{(0)} \mid \mathfrak{e}\right], k=1,2, \ldots$, we obtain by the law of large numbers,

$$
\lim _{x \rightarrow \infty} \mathbb{P}\left(\frac{1}{x \Theta_{1}} \sum_{k=1}^{[x]} \zeta_{k}^{(0)} \leq \frac{1}{x^{\varepsilon} \Theta_{1}} \mid \mathfrak{e}\right)=0 \quad \mathbb{P} \text {-almost surely. }
$$

Thus,

$$
\lim \sup _{x \rightarrow \infty} \mathbb{P}\left(\sum_{k=1}^{[x]} \zeta_{k}^{(0)} \leq x^{1-\varepsilon}\right) \leq \mathbb{E}\left[\lim \sup _{x \rightarrow \infty} \mathbb{P}\left(\sum_{k=1}^{[x]} \zeta_{k}^{(0)} \leq x^{1-\varepsilon} \mid \mathfrak{e}\right)\right]=0 .
$$

As a result, for any $\delta>0$ and all $x \geq x_{0}(\delta)$, we obtain

$$
(1-\delta) \mathbb{P}\left(A_{\mathrm{T}}>x\right) \leq \mathbb{P}\left(B_{\mathrm{T}}>x^{1-\varepsilon}\right) .
$$

To deduce an estimate for $\mathbb{P}\left(B_{\mathrm{T}}>x\right)$ from above, we write

$$
\mathbb{P}\left(B_{\mathrm{T}}>x\right) \leq \mathbb{P}\left(A_{\mathrm{T}}>x^{1-\varepsilon}\right)+\mathbb{P}\left(B_{\mathrm{T}}>x ; A_{\mathrm{T}} \leq x^{1-\varepsilon}\right) .
$$

Furthermore, letting $\hat{T}_{x}=\min \left\{k:\left\|\boldsymbol{Y}_{k}\right\|>x\right\}$, we have

$$
\mathbb{P}\left(B_{\mathrm{T}}>x ; A_{\mathrm{T}} \leq x^{1-\varepsilon}\right) \leq \mathbb{P}\left(T>x^{\varepsilon / 2}\right)+\sum_{1 \leq l \leq x^{\varepsilon / 2}} \mathbb{P}\left(\hat{T}_{x}=l ; A_{\mathrm{T}} \leq x^{1-\varepsilon}\right) .
$$

By the Markov inequality, we have

$$
\begin{aligned}
\sum_{1 \leq l \leq x^{\varepsilon / 2}} \mathbb{P}\left(\hat{T}_{x}=l ; A_{\mathrm{T}} \leq x^{1-\varepsilon}\right) & \leq \sum_{1 \leq l \leq x^{\varepsilon / 2}} \mathbb{P}\left(X_{l-1} \leq x^{1-\varepsilon} ;\left\|\boldsymbol{Y}_{l}\right\|>x\right) \\
& \leq x^{\varepsilon / 2} \mathbb{P}\left(\sum_{k=1}^{\left[x^{1-\varepsilon}\right]} \zeta_{k}^{(0)}>x\right) \\
& \leq x^{-\varepsilon / 2} \mathbb{E}\left[\left\|\boldsymbol{Y}_{1}\right\|\right] .
\end{aligned}
$$

Hence, recalling (18), we obtain $\mathbb{P}\left(B_{\mathrm{T}}>x ; A_{\mathrm{T}} \leq x^{1-\varepsilon}\right)=O\left(x^{-\varepsilon / 4}\right)$ implying, in view of (23),

$$
\mathbb{P}\left(B_{\mathrm{T}}>x\right) \leq \mathbb{P}\left(A_{\mathrm{T}}>x^{1-\varepsilon}\right)+O\left(x^{-\varepsilon / 4}\right) .
$$

Combining (22) and (24) and first letting $x \rightarrow \infty$ and then $\varepsilon \rightarrow 0$ we justify, by Lemma 5 , the equivalence

$$
\mathbb{P}\left(B_{\mathrm{T}}>x\right) \sim \mathbb{P}\left(A_{\mathrm{T}}>x\right) \sim \frac{K_{0}}{\log x} .
$$


Finally,

$$
\mathbb{P}\left(B_{\mathrm{T}}>x\right) \leq \mathbb{P}\left(L_{\mathrm{T}}>x\right) \leq \mathbb{P}\left(T B_{\mathrm{T}}>x\right) \leq \mathbb{P}\left(B_{\mathrm{T}}>x^{1-\varepsilon}\right)+\mathbb{P}\left(T>x^{\varepsilon}\right),
$$

and applying (18) and Lemma 5 proves the first equivalence in (20).

One may check (21) by similar arguments.

Corollary 1. If conditions (14) and (15) hold and $\mathbb{P}\left(\theta_{1}>0\right)=1$, then

$$
F(n):=\mathbb{E}\left[1-\exp \left\{-\sum_{i=1}^{N} L_{\mathrm{T} i} Q_{n}^{(i, N)}(\mathbf{0})\right\}\right] \sim \frac{2^{N-1} K_{0}}{\log n} \text { as } n \rightarrow \infty .
$$

Proof. Clearly,

$$
L_{\mathrm{T} 1} Q_{n}^{(1, N)}(\mathbf{0}) \leq \sum_{i=1}^{N} L_{\mathrm{T} i} Q_{n}^{(i, N)}(\mathbf{0}) \leq L_{\mathrm{T}} \sum_{i=1}^{N} Q_{n}^{(i, N)}(\mathbf{0})
$$

and, by (6),

$$
\sum_{i=1}^{N} Q_{n}^{(i, N)}(\mathbf{0}) \sim Q_{n}^{(1, N)}(\mathbf{0}) \sim c_{1} n^{-1 / 2^{(N-1)}} .
$$

To conclude the proof it remains to observe that

$$
\mathbb{E}\left[1-\mathrm{e}^{-\lambda L_{\mathrm{T}}}\right] \sim \mathbb{E}\left[1-\mathrm{e}^{-\lambda L_{\mathrm{T} 1}}\right] \sim \frac{K_{0}}{\log (1 / \lambda)} \quad \text { as } \lambda \rightarrow+0
$$

due to Lemma 6, and the Tauberian theorem [4, Chapter XIII.5, Theorem 4] applied, for instance, to the right-hand side of

$$
\lambda^{-1} \boldsymbol{E}\left[1-\mathrm{e}^{-\lambda L_{\mathrm{T}}}\right]=\int_{0}^{\infty} \boldsymbol{P}\left(L_{\mathrm{T}}>x\right) \mathrm{e}^{-\lambda x} \mathrm{~d} x,
$$

and to use the inequalities

$$
\mathbb{E}\left[1-\exp \left\{-L_{\mathrm{T} 1} Q_{n}^{(1, N)}(\mathbf{0})\right\}\right] \leq F(n) \leq \mathbb{E}\left[1-\exp \left\{-L_{\mathrm{T}} \sum_{i=1}^{N} Q_{n}^{(i, N)}(\mathbf{0})\right\}\right] .
$$

Proof of Theorem 2. We first check (16). Note that each particle of type $i$ of generation $n$ has either a parent of type 0 (of generation $n-1$ ), or an ancestor of generation $k, 1 \leq k<n$ whose parent is of type 0 ; recall that the number of particles of type $i$ of generation $k$ having a parent of type 0 is denoted by $Y_{k i}$. By a decomposition of $Z_{n i}$ based on this fact and using the branching property, we obtain

$$
\mathbb{E}\left[1-s_{1}^{Z_{n 1}} \cdots s_{N}^{Z_{n N}}\right]=\mathbb{E}\left[1-\prod_{k=1}^{n} \prod_{i=1}^{N}\left(H_{n-k}^{(i, N)}(s)\right)^{Y_{k i}}\right]=\mathbb{E}\left[1-\mathrm{e}^{R(n ; s)}\right],
$$

where $H_{0}^{(i, N)}(s)=s_{i}$ by convention, and

$$
R(n ; s)=\sum_{k=1}^{n} \sum_{i=1}^{N} Y_{k i} \log H_{n-k}^{(i, N)}(s) .
$$

In particular,

$$
\mathbb{P}\left(\boldsymbol{Z}_{n} \neq \mathbf{0}\right)=\mathbb{E}\left[1-\mathrm{e}^{R(n ; \mathbf{0})} ; T \leq \sqrt{n}\right]+O(\mathbb{P}(T>\sqrt{n}))
$$


Since $\log (1-x) \sim-x$ as $x \rightarrow+0$ and for $k \leq \sqrt{n}$ as $n \rightarrow \infty$,

$$
Q_{n}^{(i, N)}(\mathbf{0})=1-H_{n}^{(i, N)}(\mathbf{0}) \leq Q_{n-k}^{(i, N)}(\mathbf{0}) \leq Q_{n-\sqrt{n}}^{(i, N)}(\mathbf{0})=(1+o(1)) Q_{n}^{(i, N)}(\mathbf{0}),
$$

we obtain

$$
\begin{aligned}
\mathbb{E}\left[e^{R(n ; \mathbf{0})} ; T \leq \sqrt{n}\right] & =\mathbb{E}\left[\exp \left\{-(1+o(1)) \sum_{i=1}^{N} L_{n i} Q_{n}^{(i, N)}(\mathbf{0})\right\} ; T \leq \sqrt{n}\right] \\
& =\mathbb{E}\left[\exp \left\{-(1+o(1)) \sum_{i=1}^{N} L_{\mathrm{T} i} Q_{n}^{(i, N)}(\mathbf{0})\right\} ; T \leq \sqrt{n}\right] \\
& =\mathbb{E}\left[\exp \left\{-(1+o(1)) \sum_{i=1}^{N} L_{\mathrm{T} i} Q_{n}^{(i, N)}(\mathbf{0})\right\}\right]-O(\mathbb{P}(T>\sqrt{n})) .
\end{aligned}
$$

Thus,

$$
\mathbb{P}\left(\boldsymbol{Z}_{n} \neq \mathbf{0}\right)=\mathbb{E}\left[1-\exp \left\{-(1+o(1)) \sum_{i=1}^{N} L_{\mathrm{T} i} Q_{n}^{(i, N)}(\mathbf{0})\right\}\right]+O(\mathbb{P}(T>\sqrt{n})),
$$

and (16) follows from Corollary 1 and (18).

Now we prove (17). Recall that we always take $X_{0}=1, Z_{0}=\mathbf{0}$.

Consider first the $N=1$ case. Writing for simplicity $Y_{k}=Y_{k 1}, Z_{n}=Z_{n 1}, s=s_{1}$, and $H_{n}(s)=H_{n}^{(1,1)}(s)=\boldsymbol{E}\left[s^{Z_{n}} \mid Z_{0}=1\right]$, we have

$$
\mathbb{E}\left[s^{Z_{n}} \mid Z_{n}>0\right]=\frac{\mathbb{E}\left[s^{Z_{n}}\right]-\mathbb{E}\left(Z_{n}=0\right)}{\mathbb{P}\left(Z_{n}>0\right)}=1-\frac{\mathbb{E}\left[1-s^{Z_{n}}\right]}{\mathbb{P}\left(Z_{n}>0\right)},
$$

and by (26),

$$
\mathbb{E}\left[1-s^{Z_{n}}\right]=\mathbb{E}\left[1-\exp \left\{\sum_{k=1}^{n} Y_{k} \log H_{n-k}(s)\right\}\right] .
$$

By the criticality condition, $1-H_{n}(0) \sim\left(b_{1} n\right)^{-1}$. Thus, if $s=\mathrm{e}^{-\lambda /\left(b_{1} n^{t}\right)}$ then

$$
1-s \sim \frac{\lambda}{b_{1} n^{t}} \sim 1-H_{\left[n^{t} / \lambda\right]}(0),
$$

where $[x]$ denotes the integral part of $x$. Hence, it follows that for any $t>1$ as $n \rightarrow \infty$,

$$
1-H_{n}\left(\mathrm{e}^{\lambda / n^{t}}\right) \sim 1-H_{n}\left(H_{\left[n^{t} / \lambda\right]}(0)\right)=1-H_{n+\left[n^{t} / \lambda\right]}(0) \sim \frac{\lambda}{b_{1} n^{t}} .
$$

Similar to the previous estimates for the survival probability of the $(N+1)$-type branching process (recall that $\left.\left(X_{0}, Z_{0}\right)=(1,0)\right)$, we obtain

$$
\mathbb{E}\left[1-\exp \left\{-\frac{\lambda Z_{n}}{b_{1} n^{t}}\right\}\right] \sim \mathbb{E}\left[1-\exp \left\{-\lambda c n^{-t} L_{\mathrm{T} 1}\right\}\right] \sim \frac{K_{0}}{t \log n} .
$$

Since $\mathbb{P}\left(Z_{n}>0\right) \sim K_{0} / \log n$, it follows that for any fixed $t>1$ and $\lambda>0$,

$$
\lim _{n \rightarrow \infty} \mathbb{E}\left[\exp \left\{-\frac{\lambda Z_{n}}{b_{1} n^{t}}\right\} \mid Z_{n}>0\right]=1-\frac{1}{t} .
$$


This implies that the conditional law of $Z_{n} /\left(b_{1} n^{t}\right)$ given $Z_{n}>0$ converges to the law of a random variable $X$ with $\boldsymbol{P}(X=0)=1-t^{-1}$ and $\boldsymbol{P}(X=+\infty)=t^{-1}$. Therefore, for any $t>1$,

$$
\begin{aligned}
G(t) & =\lim _{n \rightarrow \infty} \mathbb{P}\left(n^{-t} Z_{n} \leq b_{1} \mid Z_{n}>0\right) \\
& =\lim _{n \rightarrow \infty} \mathbb{P}\left(\frac{\log Z_{n}}{\log n} \leq t \mid Z_{n}>0\right) \\
& =1-\frac{1}{t} .
\end{aligned}
$$

Since $\lim _{t \downarrow 1} G(t)=0$, we may write (28), for any $t>0$, as

$$
\lim _{n \rightarrow \infty} \mathbb{P}\left(\frac{\log Z_{n}}{\log n} \leq t \mid Z_{n}>0\right)=1-\frac{1}{1+\max (0, t-1)}
$$

as desired.

Now, we consider the $N \geq 2$ case and use the equality

$$
\mathbb{E}\left[s_{1}^{Z_{n 1}} \cdots s_{N}^{Z_{n N}} \mid Z_{n 1}>0\right]=\frac{\mathbb{E}\left[1-s_{2}^{Z_{n 2}} \cdots s_{N}^{Z_{n N}} 1_{\left\{Z_{n 1}=0\right\}}\right]}{\mathbb{P}\left(Z_{n 1}>0\right)}-\frac{\mathbb{E}\left[1-s_{1}^{Z_{n 1}} \cdots s_{N}^{Z_{n N}}\right]}{\mathbb{P}\left(Z_{n 1}>0\right)} .
$$

We study each term on the right-hand side of (30) separately. By (26) and $\log (1-x) \sim-x$ as $x \rightarrow+0$, we see that as $n \rightarrow \infty$,

$$
\mathbb{E}\left[1-s_{1}^{Z_{n 1}} \cdots s_{N}^{Z_{n N}}\right]=\mathbb{E}\left[1-\exp \left\{-(1+o(1)) R_{N}(n, s)\right\}\right],
$$

where

$$
R_{N}(n, s):=\sum_{k=1}^{n} \sum_{i=1}^{N} Y_{k i} Q_{n-k}^{(i, N)}(s)
$$

Now let $t_{1}, \ldots, t_{N}$ be a tuple of positive numbers satisfying (7). From Lemma 2, it follows that, for $1-s_{l}=n^{-t_{l}}, l=1, \ldots, N$,

$$
Q_{n}^{(i, N)}(\boldsymbol{s}) \asymp n^{-\min _{i \leq l \leq N}\left(t_{l}-l+i\right)}=n^{-i-\min _{i \leq l \leq N}\left(t_{l}-l\right) .}
$$

Since

$$
\min _{1 \leq i \leq N} \min _{i \leq l \leq N}\left(t_{l}-l+i\right)=\min _{1 \leq l \leq N}\left(t_{l}-l+1\right) \geq 1,
$$

by our conditions, we have as $n \rightarrow \infty$,

$$
Q_{n}^{(i, N)}(\boldsymbol{s}) \ll Q_{n}^{(1, N)}(\boldsymbol{s}) \asymp n^{-\min _{1 \leq l \leq N}\left(t_{l}-l+1\right)} .
$$

Thus, there exist constants $C_{j}, j=1,2,3,4$ such that on the set $T \leq \sqrt{n}$ the estimates

$$
C_{1} L_{\mathrm{T} 1} Q_{n}^{(1, N)}(\boldsymbol{s}) \leq R_{N}(n, s) \leq \sum_{k=1}^{n} \sum_{i=1}^{N} Y_{k i} Q_{n-k}^{(i, N)}(\boldsymbol{s}) \leq C_{2} L_{\mathrm{T}} \sum_{i=1}^{N} Q_{n}^{(i, N)}(\boldsymbol{s})
$$

are valid for all sufficiently large $n$. This, in turn, implies that

$$
C_{3} L_{\mathrm{T} 1} n^{-\min _{1 \leq l \leq N}\left(t_{l}-l+1\right)} \leq R_{N}(n, s) \leq C_{4} n^{-\min _{1 \leq l \leq N}\left(t_{l}-l+1\right)} L_{\mathrm{T}} .
$$


Using the estimates above and (25) for the selected $t_{1}, \ldots, t_{N}$ as $n \rightarrow \infty$, we obtain

$$
\mathbb{E}\left[1-\exp \left\{-R_{N}(n, s)\right\} ; T \leq \sqrt{n}\right]=\left(\frac{1}{\log n}\right)\left(\frac{(1+o(1)) K_{0}}{1+\min _{1 \leq l \leq N}\left(t_{l}-l\right)}\right)+O(\mathbb{P}(T>\sqrt{n})),
$$

which leads, on account of (18), to

$$
\lim _{n \rightarrow \infty}(\log n) \mathbb{E}\left[1-s_{1}^{Z_{n 1}} \cdots s_{N}^{Z_{n N}}\right]=\frac{K_{0}}{1+\min _{1 \leq l \leq N}\left(t_{l}-l\right)} .
$$

Thus,

$$
\lim _{n \rightarrow \infty} \frac{\mathbb{E}\left[1-s_{1}^{Z_{n 1}} \cdots s_{N}^{Z_{n N}}\right]}{\mathbb{P}\left(Z_{n 1}>0\right)}=\frac{1}{1+\min _{1 \leq l \leq N}\left(t_{l}-l\right)}<1
$$

Furthermore,

$$
\mathbb{E}\left[1-s_{2}^{Z_{n 2}} \cdots s_{N}^{Z_{n N}} 1_{\left\{Z_{n 1}=0\right\}}\right]=\mathbb{E}\left[1-\exp \left\{\sum_{k=1}^{n} \sum_{i=1}^{N} Y_{k i} \log H_{n-k}^{(i, N)}\left(0, s_{2}\right)\right\}\right] .
$$

By the definition of $H_{n}^{(i, N)}(s),(31)$, and the choice of $s_{i}, i=2, \ldots, N$, we have

$$
1-H_{n}^{(i, N)}\left(0, s_{2}\right)=1-H_{n}^{(i, N)}(s)=Q_{n}^{(i, N)}(s) \asymp n^{-\min _{i \leq l \leq N}\left(t_{l}-l+i\right)}=o\left(n^{-1}\right) .
$$

Besides, by Lemma 3,

$$
1-H_{n}^{(1, N)}\left(0, s_{2}\right)=Q_{n}^{(1, N)}\left(0, s_{2}\right) \sim c_{1} n^{-1} \text { as } n \rightarrow \infty .
$$

Hence, it follows that on the set $T \leq \sqrt{n}$,

$$
\begin{aligned}
\sum_{k=0}^{T-1} \sum_{i=1}^{N} Y_{k i} \log H_{n-k}^{(i, N)}\left(0, s_{2}\right) & =-(1+o(1)) \sum_{k=0}^{T-1} \sum_{i=1}^{N} Y_{k i} Q_{n-k}^{(i, N)}\left(0, s_{2}\right) \\
& =-(1+o(1)) \sum_{i=1}^{N} L_{\mathrm{T} i} Q_{n}^{(i, N)}\left(0, s_{2}\right)
\end{aligned}
$$

and, moreover,

$$
Q_{n}^{(1, N)}\left(0, s_{2}\right) L_{\mathrm{T} 1} \leq \sum_{i=1}^{N} L_{\mathrm{T} i} Q_{n}^{(i, N)}\left(0, s_{2}\right) \leq C_{2} Q_{n}^{(1, N)}\left(0, s_{2}\right) L_{\mathrm{T}}
$$

Now, using the same line of arguments as earlier, one may show that

$$
\lim _{n \rightarrow \infty} \mathbb{E}\left[1-s_{2}^{Z_{n 2}} \cdots s_{N}^{Z_{n N}} 1_{\left\{Z_{n 1}=0\right\}}\right] \log n=K_{0},
$$

implying that, by (16) with $N=1$,

$$
\lim _{n \rightarrow \infty} \frac{\mathbb{E}\left[1-s_{2}^{Z_{n 2}} \cdots s_{N}^{Z_{n N}} 1_{\left\{Z_{n 1}=0\right\}}\right]}{\mathbb{P}\left(Z_{n 1}>0\right)}=1 .
$$


As a result, given (7), we have

$$
G\left(t_{1}, \ldots, t_{N}\right)=\lim _{n \rightarrow \infty} \mathbb{E}\left[s_{1}^{Z_{n 1}} \cdots s_{N}^{Z_{n N}} \mid Z_{n 1}>0\right]=1-\frac{1}{1+\min _{1 \leq l \leq N}\left(t_{l}-l\right)} .
$$

Since $\lim _{\min _{1 \leq l \leq N}\left(t_{l}-l\right) \downarrow 0} G\left(t_{1}, \ldots, t_{N}\right)=0$, by the same arguments used to derive (28) and (29), we conclude that

$$
\lim _{n \rightarrow \infty} \mathbb{E}\left[s_{1}^{Z_{n 1}} \cdots s_{N}^{Z_{n N}} \mid Z_{n 1}>0\right]=1-\frac{1}{1+\max \left(0, \min _{1 \leq l \leq N}\left(t_{l}-l\right)\right)}
$$

for all positive $t_{1}, \ldots, t_{N}$, completing the proof of Theorem 2 .

\section{The case of three types}

It follows from (6) that for a strongly critical $N$-type decomposable branching process in a constant environment

$$
\boldsymbol{P}\left(\boldsymbol{Z}_{n} \neq \mathbf{0} \mid \boldsymbol{Z}_{0}=\boldsymbol{e}_{1}\right) \sim \boldsymbol{P}\left(Z_{n 1}+\cdots+Z_{n, N-1}=0, Z_{n N}>0 \mid \boldsymbol{Z}_{0}=\boldsymbol{e}_{1}\right) .
$$

Thus, given the condition $\left\{\boldsymbol{Z}_{n} \neq \mathbf{0}\right\}$, we observe in the limit as $n \rightarrow \infty$ only type $N$ particles. This is not the case for the strongly critical $(N+1)$-type decomposable branching process in a random environment. We justify this claim by considering a strongly critical branching process with three types and prove the following statement.

Theorem 3. Let $N=2$. If Assumption 2 holds then

$$
\lim _{n \rightarrow \infty} \mathbb{P}\left(\frac{\log Z_{n 1}}{\log n} \leq t_{1}, \frac{\log Z_{n 2}}{\log n} \leq t_{2} \mid Z_{n} \neq \mathbf{0}, X_{0}=1, Z_{0}=\mathbf{0}\right)=A\left(t_{1}, t_{2}\right),
$$

where

$$
A\left(t_{1}, t_{2}\right)= \begin{cases}0 & \text { if } t_{1} \in[0, \infty), 0 \leq t_{2} \leq 1, \\ 1-\frac{1}{t_{2}} & \text { if } t_{1} \in[0, \infty), 1<t_{2}<2, \\ \frac{1}{2} & \text { if } 0 \leq t_{1}<1, t_{2} \geq 2, \\ 1-\frac{1}{2} \frac{1}{\left(1+\min \left(t_{1}-1, t_{2}-2\right)\right)} & \text { if } t_{1} \geq 1, t_{2} \geq 2\end{cases}
$$

Remark 1. Since the survival probability of particles of type 0 up to moment $n$ is of the order $n^{-1 / 2}$, particles of this type are absent in the limit.

Remark 2. Since $\lim _{\min \left(t_{1}, t_{2}-1\right) \downarrow 0} A\left(t_{1}, t_{2}\right)=0$, we have in Theorem 3 a complete description of the limiting distribution for the left-hand side of (33).

Proof of Theorem 3. We have

$$
\mathbb{E}\left[s_{1}^{Z_{n 1}} s_{2}^{Z_{n 2}} \mid \boldsymbol{Z}_{n} \neq \mathbf{0}\right]=1-\frac{\mathbb{E}\left[1-s_{1}^{Z_{n 1}} s_{2}^{Z_{n 2}}\right]}{\mathbb{P}\left(\boldsymbol{Z}_{n} \neq \mathbf{0}\right)},
$$

where

$$
\mathbb{E}\left[1-s_{1}^{Z_{n 1}} s_{2}^{Z_{n 2}}\right]=\mathbb{E}\left[1-\exp \left\{\sum_{k=1}^{n} \sum_{i=1}^{2} Y_{k i} \log H_{n-k}^{(i, N)}(s)\right\}\right]
$$


Now let $1-s_{i}=n^{-t_{i}}$. If $t_{1} \geq 1$ and $t_{2} \geq 2$, then by (16) (with $N=2$ ) and (32), we have

$$
A\left(t_{1}, t_{2}\right)=1-\lim _{n \rightarrow \infty} \frac{\mathbb{E}\left[1-s_{1}^{Z_{n 1}} s_{2}^{Z_{n 2}}\right]}{\mathbb{P}\left(\boldsymbol{Z}_{n} \neq \mathbf{0}\right)}=1-\frac{1}{2} \frac{1}{1+\min \left(t_{1}-1, t_{2}-2\right)},
$$

proving Theorem 3 for $\min \left(t_{1}-1, t_{2}-2\right) \geq 0$. Observe that

$$
\lim _{\min \left(t_{1}-1, t_{2}-2\right) \downarrow 0} A\left(t_{1}, t_{2}\right)=\frac{1}{2},
$$

and, therefore, contrary to the $\mathbb{P}\left(Z_{n 1}>0\right)$ case we need to analyze in more detail the case of positive $t_{1}, t_{2}$ meeting the condition $\min \left(t_{1}-1, t_{2}-2\right)<0$.

The same as in the proof of Theorem 2 , it is necessary to obtain estimates from above and below for

$$
R_{2}(n, s)=\sum_{k=1}^{n} \sum_{i=1}^{2} Y_{k i} Q_{n-k}^{(i, 2)}(s)
$$

given $T \leq \sqrt{n}$. Observe that in view of Lemma 4 and the representation

$$
Q_{n}^{(2,2)}\left(s_{2}\right)=1-H_{n}^{(2,2)}\left(s_{2}\right) \asymp \frac{1}{n^{t_{2}}+n},
$$

we have

$$
1-H_{n}^{(1,2)}\left(s_{1}, s_{2}\right)+1-H_{n}^{(2,2)}\left(s_{2}\right) \asymp 1-H_{n}^{(1,2)}\left(s_{1}, s_{2}\right)=Q_{n}^{(1,2)}\left(s_{1}, s_{2}\right) .
$$

This, in turn, yields for $T \leq \sqrt{n}$,

$$
C_{1} Q_{n}^{(1,2)}\left(s_{1}, s_{2}\right) L_{\mathrm{T} 1} \leq R_{2}(n, s) \leq C_{2} Q_{n}^{(1,2)}\left(s_{1}, s_{2}\right) L_{\mathrm{T}} .
$$

From this estimate, (25), and Lemma 4, we obtain

$$
\mathbb{E}\left[1-s_{1}^{Z_{n 1}} s_{2}^{Z_{n 2}}\right] \sim \frac{K_{0}}{C\left(t_{1}, t_{2}\right)} \log n \quad \text { as } n \rightarrow \infty,
$$

where

$$
C\left(t_{1}, t_{2}\right)= \begin{cases}\frac{1}{2} & \text { if } t_{1} \in(0, \infty), 0<t_{2} \leq 1, \\ \frac{t_{2}}{2} & \text { if } t_{1} \in(0, \infty), 1<t_{2}<2, \\ 1 & \text { if } 0<t_{1}<1, t_{2} \geq 2, \\ 1+\min \left(t_{1}-1, t_{2}-2\right) & \text { if } t_{1} \geq 1, t_{2} \geq 2 .\end{cases}
$$

Since $\mathbb{P}\left(\boldsymbol{Z}_{n} \neq \mathbf{0}\right) \sim 2 K_{0}(\log n)^{-1}$ for $N=2$, we conclude that for positive $t_{1}$ and $t_{2}$,

$$
\begin{aligned}
\lim _{n \rightarrow \infty} \mathbb{E}\left[s_{1}^{Z_{n 1}} s_{2}^{Z_{n 2}} \mid \boldsymbol{Z}_{n} \neq \mathbf{0}, X_{0}=1, \boldsymbol{Z}_{0}=\mathbf{0}\right] & =1-\lim _{n \rightarrow \infty} \frac{\mathbb{E}\left[1-s_{1}^{Z_{n 1}} s_{2}^{Z_{n 2}}\right]}{\mathbb{P}\left(\boldsymbol{Z}_{n} \neq \mathbf{0}\right)} \\
& =1-\frac{1}{2 C\left(t_{1}, t_{2}\right)} \\
& =A\left(t_{1}, t_{2}\right) .
\end{aligned}
$$

Hence, the statement of Theorem 3 follows in an ordinary way. 


\section{Acknowledgements}

V. Vatutin was supported in part by the Russian Foundation for Basic Research (Project No. 14-01-00318) and by the French National Center for Scientific Research for a scientific stay of three months in 2013 at the Université de Bretagne-Sud, where the present work was essentially carried out. Q. Liu was supported in part by the National Natural Science Foundation of China (grants no. 11171044, no. 11571052, and no. 11401590).

\section{References}

[1] Afanasyev, V. I. (1999). On the maximum of a critical branching process in a random environment. Discrete Math. Appl. 9, 267-284.

[2] Afanasyev, V. I., Geiger, J., Kersting, G. and Vatutin, V. A. (2005). Criticality for branching processes in random environment. Ann. Prob. 33, 645-673.

[3] Athreya, K. B. And Ney, P. E. (1972). Branching Processes. Springer, New York.

[4] Feller, W. (1966). An Introduction to Probability Theory and Its Applications, Vol. II. John Wiley, New York.

[5] Foster, J. AND Ney, P. (1976). Decomposable critical multi-type branching processes. Sankhya A 38, 28-37.

[6] Foster, J. AND NEY, P. (1978). Limit laws for decomposable critical branching processes. Z. Wahrscheinlichkeitsth. 46, 13-43.

[7] Geiger, J. And Kersting, G. (2001). The survival probability of a critical branching process in a random environment. Theory Prob. Appl. 45, 517-525.

[8] Smith, W. L. and Wilkinson, W. E. (1969). On branching processes in random environments. Ann. Math. Statist. 40, 814-827.

[9] Vatutin, V., Dyakonova, E., Jagers, P. and Sagitov, S. (2012). A decomposable branching process in a Markovian environment. Internat. J. Stoch. Anal. 2012, 694285. 